\title{
THE INCIDENCE OF NEONATAL INFECTIONS (MENINGITS AND SEPSIS) DURING A 42 YEAR PERIOD, FROM 1975-2016 IN WESTERN PART OF SWEDEN
}

Margrét Johansson Gudjónsdóttir ${ }^{1,2}$, Elisabet Hentz², Anders Elfvin ${ }^{1,2}$, Ingemar Tessin², Birger Trollfors ${ }^{3}$

${ }^{1}$ Division of Neonatology, Department of Paediatrics, Institute of Clinical Sciences, Sahlgrenska Academy, Gothenburg University, Gothenburg, Sweden. ${ }^{2}$ Department of

Neonatology, Queen Silvias Children Hospital, Sahlgrenska University Hospital, Gothenburg, Sweden. ${ }^{3}$ Department of Pediatrics, Queen Silvias Children Hospital, Sahlgrenska University Hospital, Gothenburg, Sweden

\section{Background}

Both sepsis and meningitis are a major cause of morbidity and mortality of newborns. The main neonatal pathogens are Group B Streptococci (GBS) Staphylococcus Aureus (SA) and Escherichia Coli (E. Coli).

\section{Aim}

The main aim of this study is to document the incidence of early and late-onset neonatal invasive infections like sepsis and meningitis in Gothenburg, Sweden and five surrounding municipalities over a 42 year period. The study will give information of changes in mortality, morbidity, risk factors and aetiology.

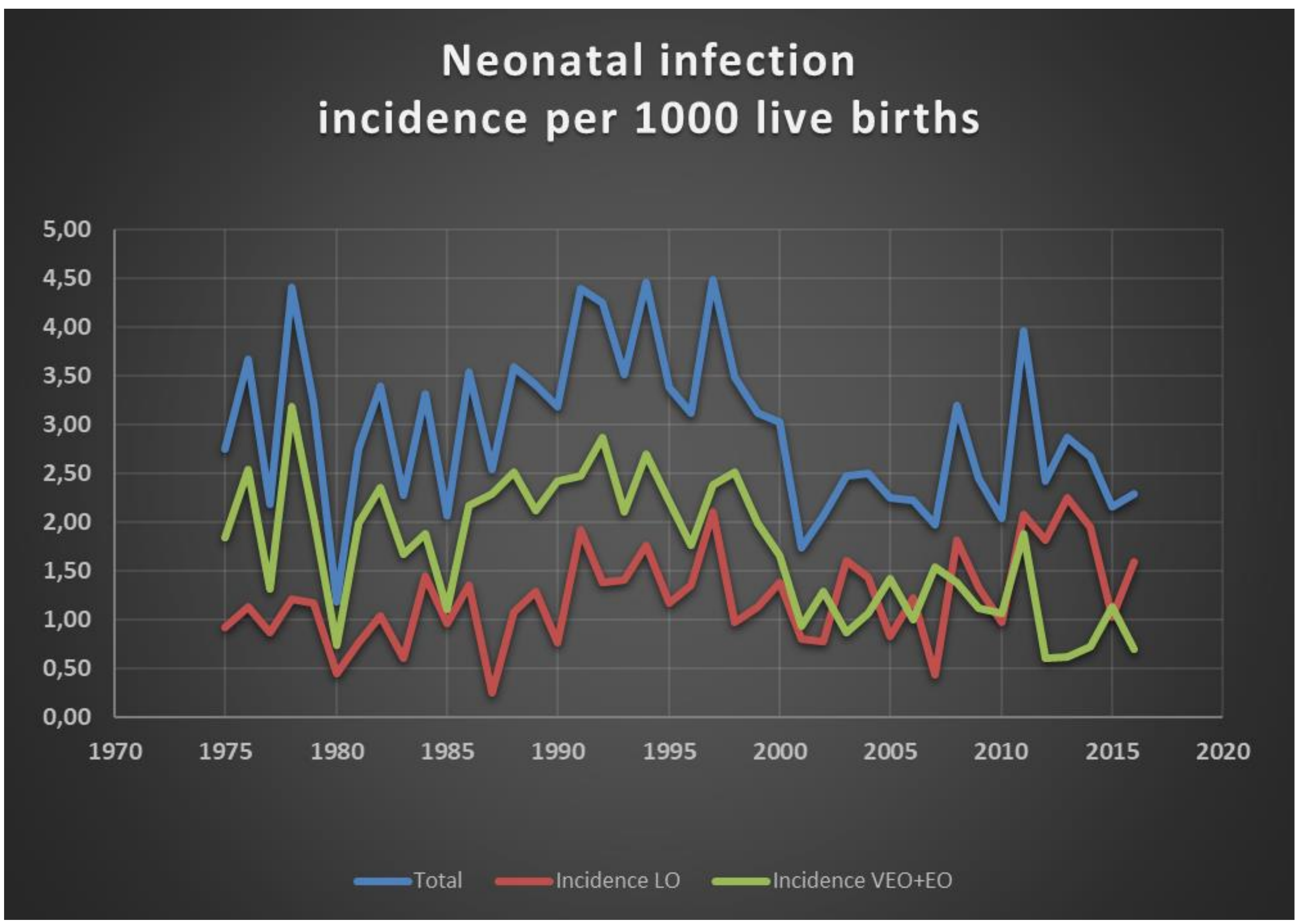

\section{Methods}

Information regarding positive culture in blood and cerebral spinal fluid (CSF) among children younger than 28 days was collected from the Clinical Microbiological Laboratory at the Sahlgrenska University Hospital which serves all hospitals in the study area. Patients

characteristics, risk factors, signs and symptoms of infection and outcome were registered. Cases of infection were classified according to infant's age at the time of test taken for culture. Very earlyonset, VEO (<24 hours of life), Early-onset, EO (1-6 days of life), and Late onset, LO (7-27 days of life).

\section{Results}

Total 1007 episodes identified in 990 infants during the study period. The mean incidence was $3.22 / 1000$ live births. GBS $22 \%$, SA $20 \%$ and E. Coli 10\%. Median age 3 days, 243 (24\%) VEO,

$332(33 \%)$ EO and 432 (43\%) LO. Male/female ratio, 572/435 (57\%/43\%). The median gestational age was 29 weeks and median birthweight $1195 \mathrm{~g}$ among the 93 patients that died as a consequence of the infection compared to 37 weeks and $2900 \mathrm{~g}$ among the patients that survived. The mortality among patients with infection decreased from $15 \%(1975-1984)$ to $6 \%(2007-2016)$.

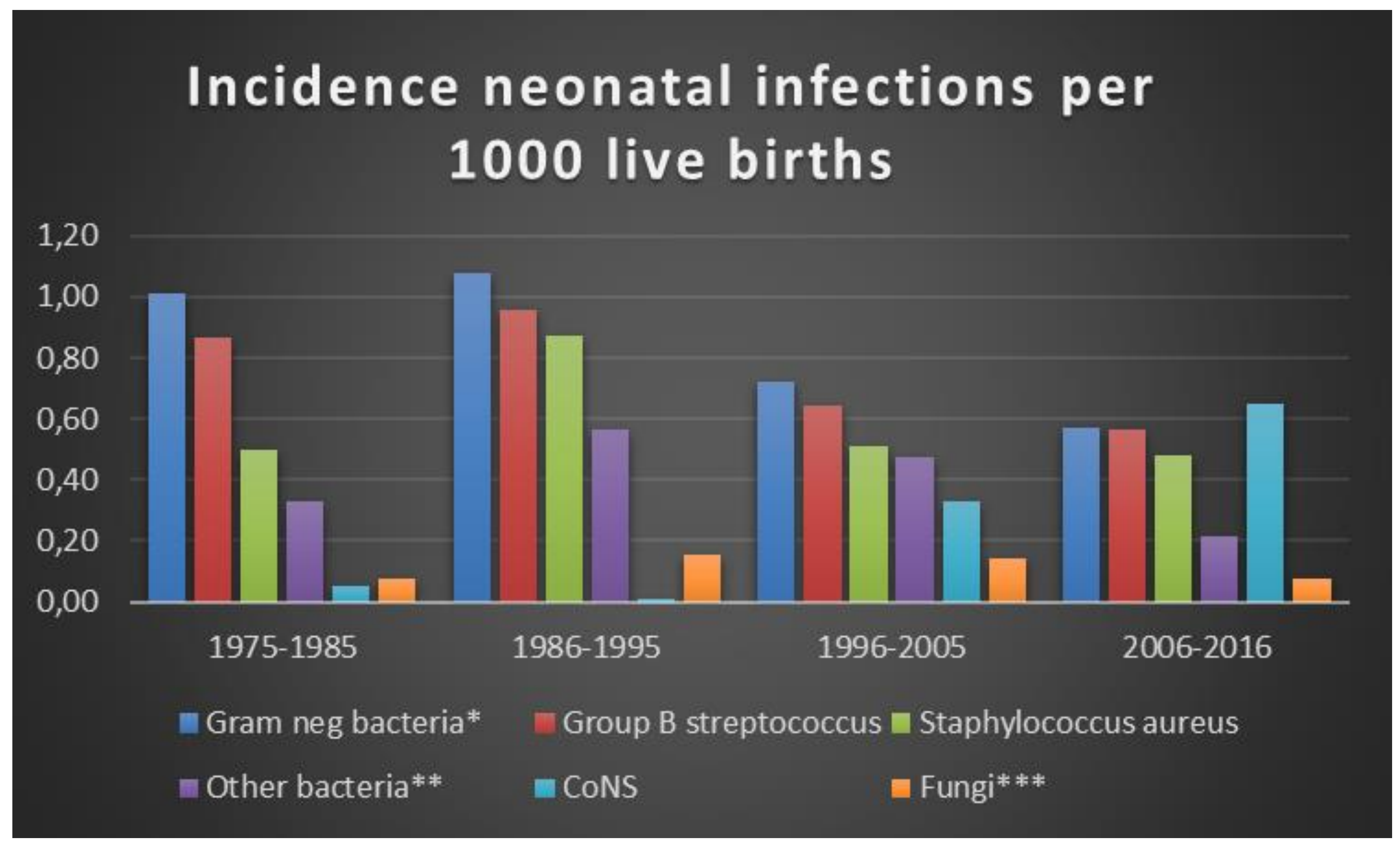

Conclusions

The trend is showing decline in VEO and EO, it could be attributable to the start of a risk-based

approach for

intrapartum antibiotics in 2008. The

premature infant is at

increased risk for

mortality and

opportunistic

infections with longer

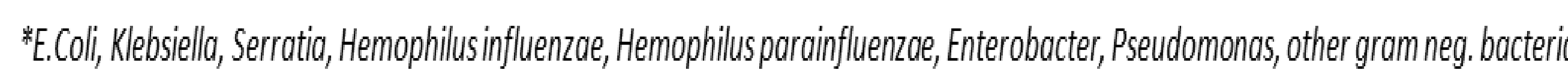
hospital stay. 